\title{
REFLEXIONES EN TORNO A LA REFORMA CONSTITUCIONAL DEL SENADO
}

\author{
FRANCISCO FERNÁNDEZ SEGADO \\ Catedrático de Derecho Constitucional \\ Universidad de Santiago de Compostela
}




\section{SUMARIO}

I. Introducción. II. Composición del Senado: 1. El concepto de "Cámara de representación territorial». 2. El sistema de elección. III. FUNCIONES DEL SENADO: 1. Función legislativa. 2. Función de control. 3. Otras funciones. 


\title{
REFLEXIONES EN TORNO A LA REFORMA CONSTITUCIONAL DEL SENADO
}

\author{
POR \\ FRANCISCO FERNÁNDEZ SEGADO \\ Catedrático de Derecho Constitucional \\ Universidad de Santiago de Compostela
}

\section{INTRODUCCIÓN}

Una reforma de la Constitución debe exigir como «prius» inexcusable de un consenso político equiparable al alcanzado en el momento constituyente. El acuerdo mayoritario que concitó nuestra "Carta Magna» es, a nuestro entender, sin ningún género de dudas, el mayor capital político de la Constitución y uno de los logros de más trascendencia, si no el que más, de la transición política y aún nos atreveriamos a decir, de toda nuestra historia política contemporánea, logro éste que adquiere aún mayor relevancia si se pone en conexión con una de las constantes del constitucionalismo histórico español: el carácter partidista de nuestras Constituciones, del que derivó sin solución de continuidad la permanente inestabilidad constitucional.

Por otra parte, a nuestro entender, la posible reforma de la Constitución debe ceñirse al Título III de la misma, en aquellos de sus preceptos que deban verse alterados como consecuencia de un nuevo diseño orgánico y funcional del Senado. En línea con ello, no creemos deba verse modificado el Título VIII. Ello no sólo introduciría un elemento de notable complejidad en la reforma, sino que dificultaría hasta el extremo, muy previsiblemente, el logro de un consenso político generalizado. Todo ello al 
margen ya de la evidencia de que el Título VIII no ha sido desarrollado en todas sus posibilidades potenciales por la vía estatutaria.

\section{COMPOSICIÓN DEL SENADO}

\section{El concepto de «Cámara de representación territorial»}

El artículo 69.1 de la Constitución concibe al Senado como «la Cámara de representación territorial». La «representación territorial» es una representación política cualificada por su referencia a los intereses generales de las entidades que integran la estructura territorial del Estado, a todas ellas, pero muy especialmente a las Comunidades Autónomas.

Basta con atender mínimamente a las funciones que el constituyente atribuyó al Senado para constatar la evidencia de la anterior reflexión. En efecto, el Senado tiene una función determinante en la apreciación de la necesidad de dictar leyes de armonización (art. 150.3 CE), en la aprobación de las medidas compulsivas a que se refiere el artículo 155.1 CE, en la celebración de convenios entre las Comunidades (art. 145.2 CE)... Ello nos debe llevar a la inequívoca conclusión de la prevalencia de los intereses territoriales comunitarios en el conjunto de actividades de la Cámara Alta.

Siendo ello así, no puede olvidarse que la «territorialidad» sólo puede hacer alusión a conjuntos sociales territorialmente definidos, y que esta condición, a la vista del artículo 137 de la Constitución, la comparten los municipios, las provincias y las Comunidades Autónomas, a lo que no puede obstar el hecho de que mientras la autonomía que se predica de las Comunidades Autónomas es política, aquella otra de la que gozan las provincias y municipios es meramente administrativa.

En definitiva, aun cuando, por lo anteriormente expuesto, parece claro que la funcionalidad de la Cámara Alta debe ser la resultante de la integración de las Comunidades Autónomas en la formación de la voluntad parlamentaria del Estado, esta prevalencia comunitaria debe conjugarse, en alguna medida, con el hecho social irrefutable de la profunda raigambre social que la provincia presenta en algunas partes del territorio nacional, como acontece con el País Vasco, Castilla-León o Andalucía. Y pensamos que el sistema de elección del Senado puede ofrecernos la fórmula para lograr aquella articulación. 


\section{El sistema de elección}

El sistema de elección que proponemos para el Senado es un sistema mixto en el que se conjuga la elección directa con la elección indirecta, de conformidad con las siguientes reglas:

1. a) Atribución a cada provincia de tres senadores, manteniendo el sistema de elección actual, esto es, un sistema mayoritario con voto limitado en el que el elector habrá de dar su voto a un máximo de dos candidatos.

2. ${ }^{\text {a) }}$ Mantenimiento del régimen diferencial derivado del hecho insular al que se refiere el artículo 69.3 de la Constitución, con la sola modificación de reducir el número de senadores que corresponde a cada una de las islas mayores de tres a dos.

3.9) Atribución a las poblaciones de Ceuta y Melilla de dos senadores, de igual forma que ahora.

4. $\left.{ }^{2}\right)$ Supresión del voto limitado en aquellas circunscripciones que elijan uno o dos senadores tan sólo, en las que el elector podrá dar su voto a tantos candidatos cuantos senadores (uno o dos) hayan de elegirse.

5.9) Atribución a las Comunidades Autónomas de un número de senadores fijo por Comunidad al que ha de añadirse un número variable en función de la población, pudiendo a tal efecto optarse por una de estas dos alternativas:

A) Atribución a cada Comunidad de dos senadores y de uno más por cada 400.000 habitantes o fracción superior a 200.000 (vid. cuadro n. 1), modelo éste con el que se alcanzan un total de 289 senadores, de ellos 158 por elección directa por circunscripciones provinciales, insulares y por las de Ceuta y Melilla, y 131 por elección indirecta por la Asamblea legislativa de la Comunidad Autónoma.

B) Atribución a cada Comunidad de tres senadores y de uno más por cada 500.000 habitantes o fracción superior a 250.000 (vid cuadro $n . .2$ ), modelo éste con el que se alcanzan un total de 287 senadores, de ellos 158 por elección directa por las circunscripciones provinciales e insulares y por Ceuta y Melilla, y 129 por elección indirecta por la Asamblea legislativa de la Comunidad Autónoma.

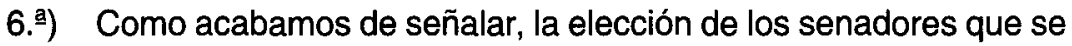
atribuyen a las Comunidades Autónomas corresponderá a la Asamblea legislativa, de conformidad con lo que establezcan los respectivos Estatutos, que 
habrán de asegurar, en todo caso, la adecuada representación proporcional. La elección habrá de recaer necesariamente en diputados autonómicos electos, bien que el mandato de senador será incompatible con el de miembro de una Asamblea legislativa autonómica, por lo que al ser elegido senador, se deberá cesar como miembro de la Asamblea, debiendo proveer el ordenamiento autonómico el modo de cubrir las vacantes producidas en las Asambleas legislativas como consecuencia de la elección de los senadores.

7.) El mandato de todos los senadores termina el día de la disolución de la Asamblea legislativa de la Comunidad Autónoma por la que fueron elegidos 0 a la que pertenecen las provincias por las que resultaron electos.

\section{CUADRO N. 1}

\begin{tabular}{|c|c|c|c|c|}
\hline \multirow{2}{*}{$\begin{array}{l}\text { Comunidad } \\
\text { Autónoma }\end{array}$} & \multirow{2}{*}{$\begin{array}{c}\text { Por } \\
\text { Provincia }\end{array}$} & \multicolumn{2}{|c|}{ Por Comunidad Autónoma } & \multirow{2}{*}{ Total } \\
\hline & & $\begin{array}{c}\text { Por } \\
\text { Población }\end{array}$ & $\begin{array}{c}\text { Por } \\
\text { Comunidad }\end{array}$ & \\
\hline Andalucía & 24 & 17 & 2 & 43 \\
\hline Aragón & 9 & 3 & 2 & 14 \\
\hline Asturias & 3 & 3 & 2 & 8 \\
\hline Baleares & 4 & 2 & 2 & 8 \\
\hline Canarias & 9 & 4 & 2 & 15 \\
\hline Cantabria & 3 & 1 & 2 & 6 \\
\hline Castilla-La Mancha & 15 & 4 & 2 & 21 \\
\hline Castilla-León & 27 & 6 & 2 & 35 \\
\hline Cataluña & 12 & 15 & 2 & 29 \\
\hline Extremadura & 6 & 3 & 2 & 11 \\
\hline Galicia & 12 & 7 & 2 & 21 \\
\hline Madrid & 3 & 12 & 2 & 17 \\
\hline Murcia & 3 & 3 & 2 & 8 \\
\hline Navarra & 3 & 1 & 2 & 6 \\
\hline País Vasco & 9 & 5 & 2 & 16 \\
\hline Rioja & 3 & 1 & 2 & 6 \\
\hline Valencia & 9 & 10 & 2 & 21 \\
\hline Ceuta & 2 & - & - & 2 \\
\hline Melilla & 2 & - & - & 2 \\
\hline TOTAL & 158 & 97 & 34 & 289 \\
\hline
\end{tabular}

(1) Un senador por cada 400.000 habitantes o fracción superior a 200.000 . 


\section{CUADRO N. 2}

\begin{tabular}{lrccr}
\hline \multirow{2}{*}{$\begin{array}{c}\text { Comunidad } \\
\text { Autónoma }\end{array}$} & $\begin{array}{c}\text { Por } \\
\text { Provincia }\end{array}$ & \multicolumn{2}{c}{ Por Comunidad Autónoma } & \multirow{2}{*}{$\begin{array}{c}\text { Por } \\
\text { Población }\end{array}$} \\
\cline { 3 - 4 } & & 14 & $\begin{array}{c}\text { Por } \\
\text { Comunidad }\end{array}$ \\
\hline Andalucía & 24 & 2 & 3 & 41 \\
Aragón & 9 & 2 & 3 & 14 \\
Asturias & 3 & 2 & 3 & 8 \\
Baleares & 4 & 3 & 3 & 9 \\
Canarias & 9 & 1 & 3 & 15 \\
Cantabria & 3 & 3 & 3 & 7 \\
Castilla-La Mancha & 15 & 5 & 3 & 21 \\
Castilla-León & 27 & 12 & 3 & 27 \\
Cataluña & 12 & 2 & 3 & 11 \\
Extremadura & 6 & 6 & 3 & 21 \\
Galicia & 12 & 10 & 3 & 16 \\
Madrid & 3 & 2 & 3 & 8 \\
Murcia & 3 & 1 & 3 & 7 \\
Navarra & 3 & 4 & 3 & 16 \\
País Vasco & 9 & 1 & 3 & 7 \\
Rioja & 3 & 8 & 3 & 20 \\
Valencia & 9 & - & - & 2 \\
Ceuta & 2 & - & - & 2 \\
Melilla & 2 & 78 & 51 & 287 \\
\hline TOTAL & 158 & & & \\
\hline
\end{tabular}

(1) Un senador por cada 500.000 habitantes o fracción superior a 250.000 .

8. ${ }^{\text {) }}$ La elección de senadores en las circunscripciones provinciales, insulares y en Ceuta y Melilla tendrá lugar de modo simultáneo a las elecciones a las Asambleas legislativas de las Comunidades Autónomas en que se integren aquellas circunscripciones.

9.a) Los Presidentes de las Comunidades Autónomas serán senadores natos. Su mandato será indelegable.

Estas reglas conducirían a un Senado integrado en total por 306 ó 308 senadores, según se aplique una u otra de las fórmulas relativas a la composición expuestas, lo que entraña, prácticamente, una equiparación, un equilibrio, entre el número de senadores elegido por sufragio universal directo y el 
de aquellos otros que se asignan a las Comunidades Autónomas, y que son designados por elección indirecta o que ostentan el mandato de senador por razón del cargo: 158 frente a 148 en un caso, y 158 frente a 150 en el otro.

En otro orden de consideraciones, es preciso significar que del «hecho diferencial's de las nacionalidades y regiones no se deducen consecuencias constitucionales de ningún tipo, omisión hecha, si así se quiere considerar, de las peculiaridades de la Disposición Transitoria 2.. de la Constitución en orden a la formalización de la iniciativa autonómica. El «hecho diferencial» justifica ciertas diferencias competenciales, pero en modo alguno la quiebra de la igualdad básica entre todas las Comunidades Autónomas en el ejercicio del derecho a la autonomía. Consecuentemente, los hechos diferenciales no tienen ninguna relevancia jurídica en la composición de la Cámara Alta.

Una de las consecuencias más relevantes que se desprenden del modelo de elección propuesto para el Senado es el carácter permanente de esta Cámara, que ya no va a poder ser disuelta por el Presidente del Gobierno, a diferencia del Congreso de los Diputados. El Senado se disolverá de modo parcial con cada disolución de la Asamblea legislativa de la Comunidad que corresponda. Por lo mismo, habrá que suprimir la disolución automática de ambas Cámaras a que alude el artículo $99.5 \mathrm{CE}$, en el supuesto de fracaso de la votación de investidura de un candidato a la Presidencia del Gobierno, disolución que habrá de circunscribirse a la Cámara Baja.

Por el contrario, parece oportuno seguir manteniendo la disolución automática de ambas Cámaras en el supuesto a que se refiere el artículo 168.1 CE, esto es, en el caso de revisión total de la Constitución, o de una reforma parcial que afecte a los Títulos especialmente protegidos. En resumen, el nuevo Senado que aquí se propone será una Cámara de carácter permanente bien que sujeta a renovaciones parciales.

Este modelo de Senado, bien alejado del modelo tipo "Consejo", como es el «Bundesrat» alemán, no posibilita otra participación de los Gobiernos de las Comunidades Autónomas en las tareas del Senado más que la que pueda canalizarse a través del Presidente de la Comunidad, que, como ya hemos apuntado, es senador nato.

\section{FUNCIONES DEL SENADO}

La clave de la reforma del Senado posiblemente radique en el logro de su especialización funcional en asuntos de indole autonómica, convirtiendo a 
la par a la Cámara Alta en una institución de diálogo entre las Comunidades Autónomas, en un foro de encuentro de todas ellas a fin de que puedan pronunciarse sobre aquellos aspectos que en el conjunto de la acción política del Estado afecten a sus competencias y al ejercicio de sus funciones, o sobre la siempre necesaria armonización de sus respectivos intereses territoriales.

Se trataría, en definitiva, de aunar la voluntad de las partes en el todo, alcanzando de esta forma una dinámica participativa multilateral, con el subsiguiente arrinconamiento de la dinámica bilateral que ha presidido hasta la fecha las relaciones entre el Estado y las Comunidades Autónomas.

Este diseño funcional del Senado no puede proyectarse tan sólo sobre el estudio de los problemas autonómicos "sub specie legis", es decir, a través del análisis de las leyes con mayor contenido o incidencia autonómicos. Ello es necesario, pero no suficiente, puesto que la creación de lo que bien podríamos denominar un "espacio institucional» hábil para tratar de aquellas cuestiones propias de un Estado compuesto que muchas veces no tienen forma legislativa, exige ir más allá de la mera especialización legislativa del Senado en proyectos de relevancia autonómica.

\section{Función legislativa}

Las reflexiones que anteceden nos dan cuenta, en todo caso, de la trascendencia de la reforma de la función legislativa de la Alta Cámara y de sus relaciones con el Congreso de los Diputados.

En unos determinados ámbitos materiales, el Senado ha de ser inexcusablemente una Cámara de primera lectura. Esos ámbitos, como es lógi$\mathrm{co}$, no pueden ser sino los que tienen un acusado interés territorial-comunitario, puesto que una de las finalidades de la reforma, como ya queda dicho, debiera ser instrumentar la presencia en el Senado de los intereses territorial-comunitarios.

Ahora bien, a la hora de proceder a delimitar las leyes autonómicas y territoriales, la reforma no debiera optar por una fórmula ambigua o general de atribución al Senado, en primera, que no única, lectura, de la legislación de interés autonómico, y tampoco nos parece válido que, como acontece en la Ley Fundamental de Bonn, las iniciativas legislativas gubernamentales hayan de someterse, en primer término, al Senado para que éste pueda, dentro de un determinado plazo, tomar posición sobre aquéllas. Por el contrario, la reforma de la Constitución debiera incorporar al artículo 88 de la 
Constitución un nuevo apartado segundo en el que habrían de precisarse los proyectos de ley que, tras su aprobación por el Consejo de Ministros, han de ser sometidos para su primera lectura al Senado.

¿Cuáles debieran ser esos proyectos?

- En primer lugar, aquellos que tuviesen por objeto la planificación de la actividad económica general o la efectividad del principio de solidaridad, al amparo de las previsiones de los artículos 131 y 138 de la Constitución, respectivamente.

El artículo 149.1.13. a CE atribuye al Estado la competencia exclusiva sobre las «bases y coordinación de la planificación general de la actividad económica». Sin embargo, recordemos que en su Sentencia 1/1982, el Tribunal Constitucional, al tratar de buscar el engarce entre el artículo 10.25 del Estatuto Vasco (que otorga competencia exclusiva a la Comunidad para la "planificación de la actividad económica del País Vasco") y el antes referido artículo 149.1.13. a, llegaría a la conclusión de que ambas competencias "exclusivas" estaban llamadas objetiva y necesariamente a ser concurrentes.

Por otra parte, los órganos centrales del Estado suelen verse tentados a utilizar los llamados títulos competenciales generales u horizontales, como es el caso claro de la planificación general de la actividad económica, títulos así llamados porque inciden o cruzan horizontalmente las restantes materias, con una clara vocación expansiva, lo que exige su modulación con vistas a que no se vacie de contenido la competencia comunitaria asentada en un título vertical. Desde esta perspectiva, la intervención del Senado como Cámara de primera lectura resulta plenamente justificada.

Por último, la planificación de la actividad económica general, entre otras finalidades, debe atender (art. 131.1 CE) a la armonización del desarrollo regional, lo que explica la intervención de las Comunidades Autónomas en la elaboración de los proyectos de planificación por el Gobierno (art. 131.2 CE) y ello debe explicar igualmente el mayor protagonismo que en esta materia debe adquirir el Senado en el plano de la función legislativa.

- En segundo lugar, aquellos proyectos que versen sobre determinadas materias contempladas por el artículo 149.1 CE, respecto de las que, en la mayor parte de los casos, se atribuye al Estado la competencia exclusiva sobre su ordenación básica. Tales materias bien podrían ser las siguientes: la legislación laboral (art. 149.1.7.. $\mathrm{CE}$ ); el fomento y coordinación general de la investigación científica y técnica (art. 149.1.15. a); las bases y coordinación general de la sanidad (art. 149.1.16.a ); la legislación básica y régimen económico de la Seguridad Social (art. 149.1.17.a); las 
bases del régimen jurídico de las Administraciones Públicas y del régimen estatutario de sus funcionarios, la legislación básica sobre contratos y concesiones administrativas y el sistema de responsabilidad de todas las Administraciones Públicas (art. 149.1.18.ㄹ); la legislación, ordenación y concesión de recursos y aprovechamientos hidráulicos cuando las aguas discurran por más de una Comunidad Autónoma, y la autorización de las instalaciones eléctricas cuando su aprovechamiento afecte a otra Comunidad o el transporte de energía salga de su ámbito territorial (art. 149.1.22.. $)$; la legislación básica sobre protección del medio ambiente, como asimismo sobre montes, aprovechamientos forestales y vías pecuarias (art. 149.1.23..$^{\mathrm{a}}$ ); las bases del régimen minero y energético (art. 149.1.25. ${ }^{\mathrm{a}}$ ); las normas básicas del régimen de prensa, radio y televisión y, en general, de todos los medios de comunicación social (art. 149.1.27.ㄹ), y la defensa del patrimonio cultural, artístico y monumental español contra la exportación y la expoliación; museos, bibliotecas y archivos de titularidad estatal (art. 149.1.28. $\left.{ }^{\mathrm{a}} \mathrm{CE}\right)$.

Como puede apreciarse a la vista de la enunciación, en la mayoria de los casos, estamos ante materias cuya ordenación básica se atribuye al Estado; con ello no se pretende sino asegurar, en aras de intereses generales superiores a los de cada Comunidad Autónoma, un "común denominador normativo" (STC 1/1982) a partir del cual cada Comunidad, en defensa del propio interés general, podrá establecer las peculiaridades que le convengan dentro de su propio marco competencial. Este sistema conduce, consecuentemente, al concurso de dos centros territoriales de poder a los efectos de la normación de una determinada materia. El Tribunal Constitucional ha hablado (STC 84/1982) en estos supuestos de que el régimen juridico de la materia de que se trate es siempre resultado de la actividad concurrente del Estado y de las Comunidades Autónomas, por lo que bien puede sostenerse que dicho régimen presenta un carácter bifronte que imposibilita calificarlo, de forma unívoca, como «intracomunitario" o "extracomunitario».

Por todo lo expuesto, es evidente que en cuanto la ordenación estatal de lo que se entiende como «básico» está, al unísono, delimitando el marco de las opciones propias del poder político de las Comunidades Autónomas, parece claro que el Senado puede desempeñar una función de la mayor trascendencia en este delicado punto de interconexión entre el ordenamiento estatal y el autonómico.

Pero es que, además, si se repasan las materias seleccionadas, se verá que, en unos casos, tales materias tienen una decisiva importancia 
para los intereses comunitarios; pensemos, por ejemplo, en la regulación de los aprovechamientos hidráulicos; mientras que en otros casos (como la legislación laboral, la Seguridad Social, los museos, bibliotecas y archivos de titularidad estatal...) es la propia Norma Suprema la que determina que la ejecución o gestión habrá de corresponder a las Administraciones autonómicas.

- En tercer termino, los proyectos de ley que acojan las delegaciones legislativas a que se refiere el artículo 150.1 de la Constitución, los proyectos de leyes de armonización de las disposiciones normativas de las Comunidades Autónomas a que alude el artículo $150.3 \mathrm{y}$, finalmente, los proyectos de leyes orgánicas de transferencia o delegación en las Comunidades Autónomas de facultades correspondientes a materia de titularidad estatal, contemplados por el artículo 150.2. La aprobación, modificación o derogación de estos últimos proyectos exigirá mayoría absoluta del Senado en una votación final sobre el conjunto del proyecto.

Estamos en presencia de tres supuestos de modificación extra-estatutaria del régimen de competencias de las Comunidades Autónomas, circunstancia que es posible por cuanto, como ha reiterado el Tribunal Constitucional (así, en la STC 76/1983), la reserva que la Constitución hace al Estatuto en esta materia no es total o absoluta.

De esta forma, eventualmente, la competencia estatutaria puede ser ampliada mediante leyes "ad hoc" dictadas al amparo de las previsiones del artículo 150.1 y 2 , y su ejercicio por las distintas Comunidades armonizado mediante una norma de la naturaleza de las previstas por el artículo 150.3.

Los mecanismos regulados por el artículo 150 responden a la conveniencia de flexibilizar el sistema de distribución de competencias, pudiendo incidir sobre él, caso de que así se considerase necesario, sin necesidad de tener que acudir al mucho más rígido procedimiento de la reforma estatutaria.

A la vista de los contenidos de los proyectos de ley a que alude el artículo 150 y de la finalidad de los mismos, parece una evidencia incontestable que sea la Cámara Alta, a la que se confía el cuidado de los intereses político-territoriales comunes al conjunto de las Comunidades Autónomas, la que conozca en primera lectura de los mismos.

- En cuarto lugar, la aprobación y reforma de los Estatutos de Autonomía debe corresponder en primer término al Senado, a la Cámara en la que los intereses territorial-comunitarios han de ser tenidos por prevalentes 
en el conjunto de sus actividades. Es tan evidente esta circunstancia que, a nuestro juicio, no requiere de mayor argumentación.

- En quinto término, el Senado debe conocer en primera lectura de aquellos proyectos de ley que contengan una declaración de interés general legitimadora de la acción estatal.

El artículo 149.1.20. " atribuye al Estado la competencia exclusiva en materia de puertos y aeropuertos de interés general. Y el artículo 149.1.24. $\stackrel{\text { a }}{ }$ hace otro tanto respecto de las obras públicas de interés general. Quiere ello decir que la «declaración de interés general» hecha por el propio Estado es un título habilitante para la atribución de competencias, que no sólo incide en el marco del reparto competencial, sino que tiene una extraordinaria trascendencia en el ámbito de la financiación por cuanto, como fácilmente se comprende, la declaración de interés general comporta el que, por ejemplo, las obras sean sufragadas por el Estado y no por la Comunidad Autónoma. Pensemos, ejemplificativamente, en el Plan Director de Infraestructuras o en el Plan Hidrológico Nacional.

El «interés general» es un concepto jurídico indeterminado y la fijación de los criterios sobre lo que deba ser el «interés general» se lleva a cabo sin intervención directa de las Comunidades Autónomas, lo que ha conducido a que el Tribunal Constitucional se vea obligado a precisar los criterios y contenidos materiales del interés general supraautonómico.

Por todo ello, parece lógico que deba ser la Cámara de representación territorial la que proceda, en primer término, a determinar el «interés general» en cuanto elemento legitimador de la acción estatal en el ámbito competencial de las Comunidades Autónomas. Quizá de esta forma puedan evitarse las inconcreciones o la falta de criterios lógicos de ciertas concreciones materiales a las que se ha atendido para delimitar lo que ha de entenderse por «interés general» (pensemos, por poner un ejemplo, en la Ley 27/1992, de Puertos del Estado y de la Marina Mercante).

- En sexto y último término, el Senado debiera conocer en primer término de aquellos proyectos de leyes orgánicas mediante los que se pretenda autorizar la celebración de tratados por los que se atribuya a una organización o institución internacional el ejercicio de competencias derivadas de la Constitución, al amparo de lo previsto en el artículo 93 CE.

Es evidente que un tratado de esta naturaleza puede incidir en el sistema de distribución de competencias entre el Estado y las Comunidades Autónomas, y ello es razón más que suficiente para defender la intervención en primera lectura del Senado. 
En relación a los proyectos de ley de los que ha de conocer en primera lectura el Senado, como acaba de quedar dicho, su intervención es en primera lectura, pero no en única lectura. Quiere ello decir que de tales proyectos ha de conocer necesariamente el Congreso en segunda lectura. $Y$ a la inversa, el Senado, a nuestro entender, no debe ver circunscrita su función legislativa al examen de aquellos proyectos de los que ha de conocer en primera lectura. Bien al contrario, el Senado no debe renunciar a las competencias que en la actualidad le corresponden como Cámara de segunda lectura.

En cuanto que ambas Cámaras han de intervenir en todos los proyectos de ley, bien en primera o segunda lectura, la cuestión que de inmediato se plantea es la relativa a la resolución de las discrepancias entre una y otra. La solución a adoptar, a nuestro juicio, no puede ser idéntica, sino que dependerá de la Cámara que conoce en primer término.

- Cuando sea el Congreso quien conozca en primera lectura de un proyecto de ley, el procedimiento a seguir será el actualmente previsto por el artículo 90 de la Constitución.

- Cuando el Senado intervenga como Cámara de primera lectura, el procedimiento a seguir será el siguiente:

Aprobado el proyecto o proposición de ley por el Senado, su Presidente dará inmediata cuenta del mismo al Presidente del Congreso, el cual lo someterá a la deliberación de éste, que se acomodará al procedimiento reglamentariamente previsto al efecto.

En caso de discrepancia entre los textos aprobados por una y otra Cámara, se intentará obtener el acuerdo por una Comisión Mixta compuesta de igual número de Diputados y Senadores. La Comisión presentará un texto que será votado por ambas Cámaras. Si se tratare de un proyecto o proposición de ley orgánica, la aprobación deberá hacerse por la mayoría absoluta de los miembros de las dos Cámaras.

La fórmula que se propone entraña que aun en aquellos supuestos en que el Senado actúe como Cámara de primera lectura, en ningún caso pueda tener la última palabra desde el punto de vista legislativo, bien que tampoco su voluntad pueda ser suplida por el Congreso, requiriéndose inexcusablemente de la conjunción de voluntades de ambas Cámaras, en cuanto que el texto aprobado por la Comisión Mixta habrá de ser votado favorablemente por Congreso y Senado. En su defecto, el texto no podrá considerarse aprobado.

Si algún Decreto-ley versare sobre materias de las que ha de conocer el Senado en primera lectura, su convalidación o, en su caso, derogación, 
corresponderá tan sólo al Senado, habiendo de seguirse al efecto un procedimiento análogo al que contempla el artículo 86.2 de la Constitución.

Otra cuestión de interés en relación con la función legislativa es la atinente a la iniciativa legislativa. En este punto entendemos que el Congreso y el Senado deberán ver circunscrita su iniciativa legislativa a aquellas materias respecto de las cuales haya de iniciarse la tramitación de los proyectos de ley gubernamentales en la propia Cámara.

Finalmente, en cuanto al posible derecho de veto a una iniciativa legislativa por parte de una Comunidad Autónoma, no nos mostramos partidarios en modo alguno del reconocimiento de tal posibilidad constitucional, ni tan siquiera en el supuesto de que los efectos de dicho veto se limitaran temporalmente. La reacción por parte de los órganos autonómicos frente a un texto legal no puede ser sino la presentación de un recurso de inconstitucionalidad ante el Tribunal Constitucional, cuando consideren que el texto en cuestión afecta a su propio ámbito de autonomía y es contrario a la Constitución, pero no cabe institucionalizar un derecho de veto frente a la actuación de los órganos representativos que actualizan permanentemente la voluntad soberana del pueblo.

\section{Función de control}

La actividad de control no supone, rigurosamente considerada, más que la confrontación de una determinada conducta con el parámetro al que debe adecuarse y no incluye en si misma, por tanto, medida sancionadora alguna, aunque, como consecuencia del control, puedan accionarse los mecanismos reparadores o sancionadores existentes. A nuestro entender, en nuestra Constitución, el control parlamentario no presupone ineludiblemente la necesidad de una medida sancionadora.

Ello no debe entenderse en el sentido de que el control tenga un único significado. Bien al contrario, presenta significaciones bien diversas. En unos casos, con un sentido limitado, el «control» significa verificación o inspección de la actividad que el Gobierno realiza; en otros implica un verdadero poder de autorizar o impedir, 0 , si se prefiere, de aprobar o vetar un acto gubernamental; por último, hay supuestos en los que, como consecuencia de poderes de verificación o veto, el control significa una participación en una actividad realizada por el Gobierno. Esta última manifestación de la actividad de control ha adquirido tal importancia que, por así decirlo, 
ha cobrado vida propia, desgajándose de la función que nos ocupa y pasando a constituir la que se ha dado en llamar función de impulso o dirección política.

Entre las medidas de control que tienen un carácter limitado de inspección o verificación, el Senado podrá seguir operando a través de las preguntas e interpelaciones parlamentarias y de las Comisiones de investigación, en el bien entendido de que, por ejemplo, respecto de estas últimas, creemos que sería conveniente delimitar el objeto de la investigación que puede justificar su creación, ya que si bien parece lógico que el Congreso pueda crear una Comisión de esta naturaleza "sobre cualquier asunto de interés público», la especialización funcional del Senado debiera tener su reflejo también en este aspecto.

En cuanto a aquella manifestación del control que se traduce en una autorización parlamentaria, esto es, en una aprobación o en un veto, como ya dijimos, el Senado debiera quedar habilitado para convalidar, o en su caso derogar, aquellos Decretos-leyes que versen sobre materias de las que ha de conocer la Alta Cámara en primera lectura.

En definitiva, la actuación del Senado como órgano de control ha de tener como punto focal el conjunto de actuaciones gubernamentales relacionadas con los intereses generales de las entidades que integran la estructura territorial del Estado. Por el contrario, creemos que la Alta Cámara debe seguir apartada de toda intervención en los mecanismos de control que llevan aparejada la sanción de la responsabilidad política gubernamental.

\section{Otras funciones}

El papel del Senado en la cooperación interterritorial debe ser fortalecido. A este respecto, la primera modificación que habría de hacerse en la Constitución sería la modificación del artículo 74.2, por cuanto resulta del todo inaceptable que en el procedimiento para la aprobación de los acuerdos de cooperación entre Comunidades Autónomas tenga la última palabra el Congreso de los Diputados. A nuestro modo de ver, debiera establecerse aquí una fórmula similar a la que hemos propuesto para la solución de discrepancias entre Congreso y Senado en aquellos casos en que el Senado interviene como Cámara de primera lectura, lo que entrañaría la ineludibilidad de una convergencia de voluntades de ambas Cámaras para la aprobación de un acuerdo de cooperación intercomunitario. 
Al margen ya de lo anterior, es evidente que análoga determinación debe regir respecto del artículo 158.2, en cuanto se refiere a la distribución de los recursos del Fondo de Compensación por las Cortes Generales entre las Comunidades Autónomas.

Sería asimismo positivo en orden al mejor cumplimiento de sus funciones el establecimiento de una cierta vinculación de la Alta Cámara con los órganos multilaterales de cooperación, como es el caso del Consejo de Política Fiscal y Financiera de las Comunidades Autónomas, contemplado por el artículo 39 de la Ley Orgánica 8/1980, de Financiación de las Comunidades Autónomas, si bien esta vinculación quizá pudiera quedar plasmada con más propiedad por la vía legislativa ordinaria 0 , alternativamente, por la vía del Reglamento de la Alta Cámara.

Algo análogo podría decirse respecto de la posible creación y funcionamiento de una Conferencia de Presidentes. El artículo 4.. de la Ley 12/1983, del Proceso Autonómico, posibilitó la existencia, más de hecho y por aceptación recíproca que por una obligación jurídica específica, de las Conferencias Sectoriales integradas por los Consejeros de las diferentes Comunidades Autónomas y por el Ministro o Ministros del ramo. Pues bien, la creación de una Conferencia de Presidentes, integrada por los Presidentes autonómicos y por el Presidente del Gobierno y quizá asimismo por su Vicepresidente, podría actuar como instancia de culminación de las actuales Conferencias Sectoriales, cumpliendo funciones de la mayor relevancia y entre ellas:

a) Actuar como instancia deliberante, como foro de diálogo y debate del que bien podrían surgir las líneas generales de la política autonómica, ejerciendo de esta forma una función de auténtica dirección política en este ámbito.

b) Supervisar y dirigir la acción de las Conferencias Sectoriales.

c) Pronunciarse acerca de los proyectos normativos de la Unión Europea, pudiendo asimismo coordinar las actuaciones relacionadas con la defensa del Estado Español ante el Tribunal de Justicia de las Comunidades Europeas.

El hecho de que los Presidentes de las Comunidades Autónomas sean senadores natos y la específica relevancia del Senado en cuanto atañe a la integración de los intereses territoriales-comunitarios en la formación de la voluntad del Estado, quizá hicieran aconsejable algún tipo de vinculación de la Cámara Alta con la Conferencia de Presidentes. 
Y algo análogo podría postularse respecto de las Comisiones Coordinadoras a las que se refiere el artículo 24 de la Ley 30/1983, reguladora de la cesión de tributos del Estado a las Comunidades Autónomas, bien que estas Comisiones respondan a un modelo de relaciones bilaterales, que no multilaterales.

No creemos por el contrario que deba alterarse la intervención actual del Senado en relación con el nombramiento de miembros de otros órganos constitucionales, ni tampoco respecto del Presidente del Gobierno.

Tampoco entendemos necesario que se cree un mecanismo específico de arbitraje previo a la intervención del Tribunal Constitucional. En el orden legislativo, la paridad que se establecería entre Congreso y Senado, de acuerdo con el modelo que proponemos, en la tramitación de aquellos proyectos de los que, por su especial incidencia en el ámbito autonómico, deba conocer en primer término el Senado, impediría muy posiblemente la repetición de circunstancias como las que rodearon, por ejemplo, a la tramitación de la Ley de Costas, masivamente considerada inconstitucional por las Comunidades Autónomas y que, pese a ello, continuó su itinerario legislativo sin que fueran escuchadas en sede legislativa las argumentaciones esgrimidas por las Comunidades en cuestión. $Y$ en cuanto a los conflictos de competencias, no creemos lógico que intervenga como árbitro, previamente a la intervención del Tribunal Constitucional, el Senado.

Por último, en cuanto que el Senado ha de ser un lugar de encuentro entre el Estado y las Comunidades Autónomas en todo tipo de materias, ello debe comprender las relaciones entre uno y otras respecto a la Unión Europea. Es obvio que el Estado autonómico exige la participación de las Comunidades Autónomas en la formación de la voluntad del Estado. La posición negociadora del Estado ante la Unión Europea precisa contar con un componente autonómico, realmente esencial cuando de lo que se trata es de negociar materias de la competencia exclusiva de las Comunidades. Aquí, el Senado debiera propiciar la coordinación de las voluntades comunitarias a fin de alcanzar la voluntad estatal. También podria desempeñar un papel destacado en la concertación entre el Gobierno del Estado y los Ejecutivos autonómicos, quizá a través de la vía de la Conferencia de Presidentes, tanto en lo concerniente a la defensa de los intereses autonómicos y generales ante los organismos propios de la Unión como en lo relativo a la ejecución del Derecho Comunitario. Todo ello al margen ya del funcionamiento de la Conferencia Sectorial para 
asuntos relacionados con las Comunidades Europeas, encuadrada en el Ministerio para las Administraciones Públicas. 